\title{
Acute Maxillary Sinusitis Associated with Internal Sinus Lifting: Report of a Case
}

Alper Alkana, DDS, PhD

Nükhet Celebib, DDS

Burcu Bas ${ }^{b}$ DDS

\section{ABSTRACT}

Sinus floor augmentation (SFA) is one of the techniques that has been proposed for improving the long-term retention of dental implants. The procedure involves the creation of a submucoperiosteal pocket in the floor of the maxillary sinus for placement of a graft consisting of autogenous, allogenic, or alloplastic material. Complications of the SFA predominantly consist of disturbed wound healing, hematoma, sequestration of bone, and transient maxillary sinusitis. In this report, we presented an acute maxillary sinusitis complication following internal sinus lifting in a patient with chronic maxillary sinusitis. (Eur J Dent 2008;2:69-72)

Key words: Sinus floor augmentation; Maxillary sinusitis; Internal sinus lifting.

\section{INTRODUCTION}

Sinus floor augmentation (SFA) is one of the techniques that have been proposed for improving the long-term retention of dental implants. ${ }^{1}$ The procedure involves the creation

- Associate Professor, Department of Oral and Maxillofacial Surgery, Faculty of Dentistry, Erciyes University, Kayseri, Turkey.

b Research Assistant, Department of Oral and Maxillofacial Surgery, Faculty of Dentistry, Ondokuz Mayıs University, Samsun, Turkey.

- Corresponding author: Nükhet Celebi Ondokuz Mayis Üniversitesi, Diș Hekimliḡi Fakültesi 55139 Kurupelit / Samsun / TURKEY

Tel: +90 $3624576000-3480$

Fax: +903624576032

E-mail: nukhRhotmail.com of a submucoperiosteal pocket in the floor of the maxillary sinus for placement of a graft consisting of autogenous, allogenic, or alloplastic material. ${ }^{2}$ Currently, two main approaches to the SFA procedure can be found in the literature. These include lateral window (external) and osteotome (internal) procedures. ${ }^{3}$ External technique allows for a greater amount of bone augmentation to the atrophic maxilla but requires a larger surgical access. ${ }^{4}$ However, internal technique is considered to be a less invasive alternative to the external method to increase the volume of bone in the posterior maxilla. ${ }^{5}$

Complications of the SFA predominantly consist of disturbed wound healing, hematoma, sequestration of bone, and transient maxillary 
sinusitis. ${ }^{6}$ The last complication was considered to be the major drawback of this procedure. ${ }^{7}$ Previous investigations have reported maxillary sinusitis up to $20 \%$ of patients after SFA. ${ }^{8}$ Postoperative acute maxillary sinusitis may cause implant and graft failures. The reported cases of maxillary sinusitis developed after the lift procedure are all associated with the external techniques. On the contrary, internal procedure appears to be a safer method with rare complications.

In this report we presented an acute maxillary sinusitis complication following internal sinus lifting in a patient with chronic maxillary sinusitis. In our knowledge, this complication after internal sinus lifting procedure has not been reported in the literature.

\section{CASE REPORT}

A 52 year-old woman with chronic maxillary sinusitis was referred to our clinic for implant therapy. Clinical and radiographic examination showed no signs of acute sinusitis (Figure 1). The patient had a history of an acute sinusitis attack 6 weeks ago.

Maxillary sinus floor was augmented by means

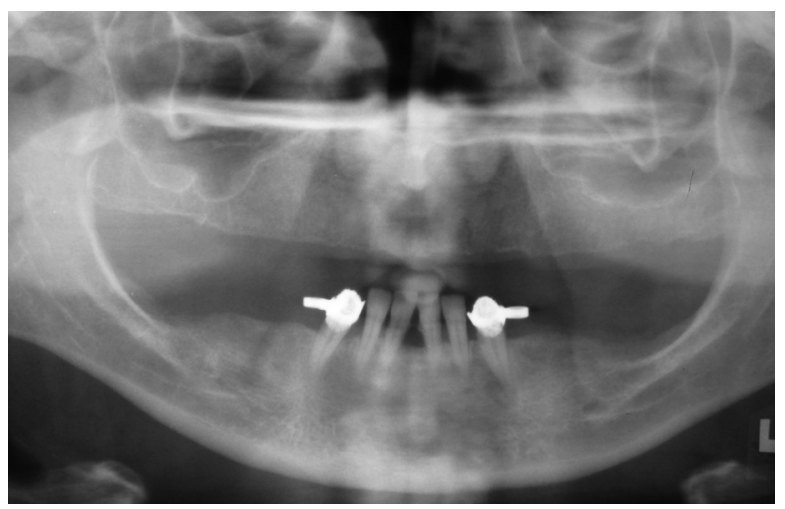

Figure 1. Preoperative radiograph of the patient.

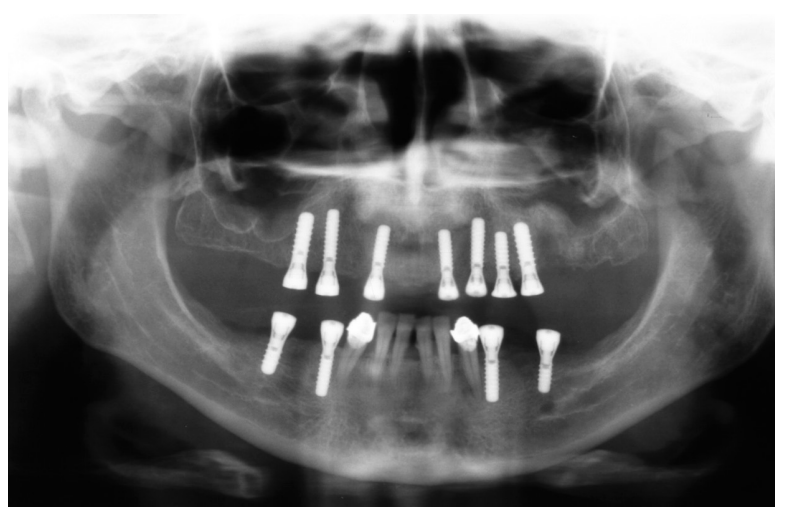

Figure 3. The insertion of a new implant to the canine region. of internal technique in the first molar region on the left side using $0.5 \mathrm{gr}$ xenograft (BioOss ${ }^{\circledR}$, Geistlich Sons Ltd) and an implant in a diameter of $4.1 \times 12 \mathrm{~mm}$ (ITI®, Straumann) was placed (Figure 2). No complications occurred during the surgical procedure. Four weeks after the surgery, the patient had pain on the region of the implant inserted with the internal lifting procedure. Clinical examination showed postnasal drip, swelling and hyperemia on the operated side. Any signs and symptoms of oro-antral communication were observed. Full opaque appearance of left maxillary sinus on the panoramic radiograph confirmed the acute maxillary sinusitis. Antibiotics (Amoxicillinclavulanate 1g, 2 times dailyl continued for 10 days in combination with a nasal decongestant (Pseudoephedrin hydrochlorur $60 \mathrm{mg}$ once a day). Although the signs of acute sinusitis were reduced after medical treatment, the pain had not ceased. Finally, the implant was extracted and a purulant fluid was drained from the implant socket. A new implant in a diameter of $4.1 \times 12 \mathrm{~mm}$ (ITIß, Straumann) was inserted to the canine region (Figure 3). Final restoration was reconstructed 4 months later. All complaints had ceased and all

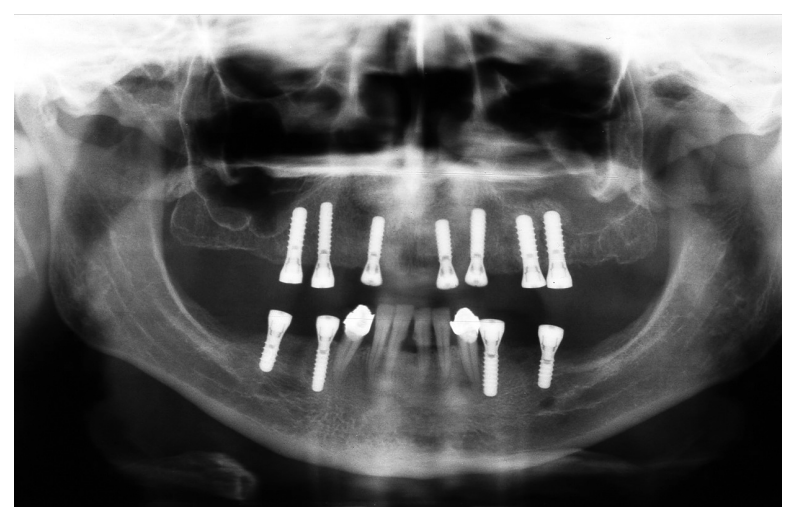

Figure 2. Post operative radiographic view of the full opaque appearance of left maxillary sinus.

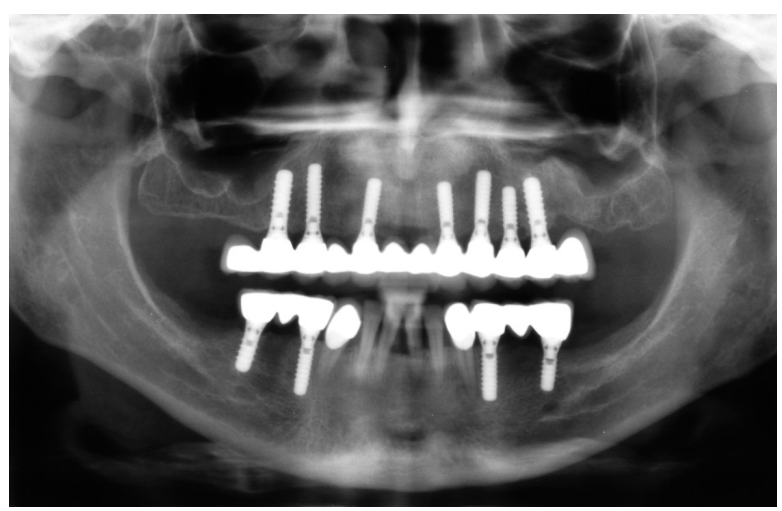

Figure 4. Radiographic view, 9 months after the operation. 
implants had a good stability at 9-month evaluation (Figure 4).

\section{DISCUSSION}

Internal sinus lifting was proposed for implant sites with at least $5-6 \mathrm{~mm}$ of bone between the alveolar crest and the maxillary sinus floor. ${ }^{9}$ For this purpose, osteotomes are used to prepare the implant site either with or without graft materials. The grafting techniques are used when the bone height is insufficient to provide stability for the implant placement. However, it has the disadvantage of loss of the graft material within the sinus leading to a sinusitis in cases of sinus membrane perforation. ${ }^{10}$

Preoperative sinus disease has been positively correlated with the development of acute postoperative sinusitis after maxillary sinus grafting. ${ }^{11}$ The maxillary sinus physiology is affected by the altered anatomic relation of the antral floor in combination with a bulging or injured surface of the lifted sinus mucosa. In addition to altered anatomy, postoperative swelling and a hematoma or seroma that fills up the maxillary sinus may also lead to reduction of the patency of the osteo-meatal unit which plays a key role in the development of sinusitis. ${ }^{7}$ In the patients with chronic sinusitis, altering the vulnerable physiology of chronic infected maxillary sinus by damaging the delicate maxillary mucosal lining with the surgical intention may be the possible cause of postoperative acute sinusitis.

Internal sinus lift procedure has the advantage of the protection of the intraosseous vessels in the maxilla and less postoperative morbidity. ${ }^{12}$ It seems to be a less invasive method with minimal risk of sinus membrane perforation. From this point of view, chronic sinusitis was not considered as a risk factor for the procedure in our case. Although the sinus membrane perforation was not observed and the graft exhibited a dome-shaped opacity in the postoperative panoramic radiograph, we encountered with an acute sinusitis attack which caused the failure of implant 1 month after the surgery.

An acute postoperative maxillary sinusitis may hazard the survival of the implants and graft. However, Kahnberg et al ${ }^{13}$ reported successful outcomes in the patients with mucosal thickening in the maxillary sinus before the augmentation of the sinus floor, as far as implant failures. Others have previously reported the successful healing of maxillary sinusitis associated with the augmentation procedure after treatment with decongestants and antibiotics in patients with a predisposition for sinusitis. ${ }^{14}$ In the present case, signs of the acute disease were reduced with medical treatment, whereas the symptoms remained.

\section{CONCLUSIONS}

Maxillary sinusitis is an inevitable complication of maxillary sinus augmentation in patients with a history of maxillary sinus disease. In these cases, implant failures may occur in the long term followup. Although internal sinus lifting is less invasive than the external technique, both procedures can damage the maxillary sinus mucosa leading to maxillary sinusitis. Therefore, careful clinical and radiographic evaluation is essential before and after the augmentation.

\section{REFERENCES}

1. Butz SJ, Huys LWJ. Long-term success of sinus augmentation using a synthetic alloplast: a 20 patients, 7 years clinical report. Implant Dent 2005;14:36-42.

2. Doud Galli SK, Lebowitz RA, Giacchi RJ, Glickman R, Jacobs JB. Chronic sinusitis complicating sinus lift surgery. Am J Rhinol 2001;15:181-186.

3. McDermott NE, Chuang SK, Woo VV, Dodson TB. Maxillary sinus augmentation as a risk factor for implant failure. Int J Oral Maxillofac Implants 2006;21:366-374.

4. Woo I, Le BT. Maxillary sinus floor elevation: Review of anatomy and two techniques. Implant Dent 2004;13:28-32.

5. Toffler M. Minimally invasive sinus floor elevation procedures for simultaneous and staged implant placement. N Y State Dent J 2004;70:38-44.

6. Timmenga NM, Raghoebar GM, van Weissenbruch $R$, Vissink A. Maxillary sinusitis after augmentation of the maxillary sinus floor: a report of 2 cases. J Oral Maxillofac Surg 2001;59:200-204.

7. Timmenga NM, Raghoebar GM, Liem RSB, van Weissenbruch R, Manson WL, Vissink A. Effects of maxillary sinus floor elevation surgery on maxillary sinus physiology. Eur J Oral Sci 2003;111:189-197.

8. Timmenga NM, Raghoebar GM, Boering G, van Weissenbruch R. Maxillary sinus function after sinus lifts for the insertion of dental implants. J Oral Maxillofac Surg 1997;55:936-939.

9. Bragger U, Gerber C, Joss A, Haenni S, Meier A, Hashorva 
E, Lang NP. Patterns of tissue remodeling after placement of ITIs dental implants using an osteotome technique: a longitudinal radiographic case cohort study. Clin Oral Impl Res 2004;15:158-166.

10. Kasabah S, Kruk J, Simunek A, Lecaro MC. Can we predict maxiller sinus mucosa perforation? Acta Med 2003;46:1923.

11. Tidwell JK, Blijdorp PA, Stoelinga PJW, Brouns JB, Hinderkers F. Composit grafting of the maxillary sinus for placement of endosteal implants. A preliminary report of 48 patients. Int J Oral Maxillofac Surg 1992;21:204-209.

12. Baumann A, Ewers R. Minimally invasive sinus lift. Limits and possibilities in the atrophic maxilla. Mund Kiefer Gesichtschir 1999;3(Suppl 1):S70-73.

13. Kahnberg KE, Ekestubbe A, Gröndahl K, Nilsson P, Hirsch JM. Sinus lifting procedure. I. One-stage surgery with bone transplant and implants. Clin Oral Impl Res 2001;12:479487.

14. Raghoebar GM, Batenburg RHK, Timmenga NM, Vissink A. Morbidity and complications of bone grafting of the floor of the maxillary sinus for the placement of endosseous implants. Mund Kiefer Gesichtschir 1999;3:65-69. 